\title{
Brotes de leptospirosis humana en la provincia de Ciego de Ávila, Cuba
}

\author{
Human leptospirosis outbreak in the district of Ciego de Ávila, Cuba \\ Miguel Suárez Hernóndez, Raydel Martínez Sánchez, Pedro €. Posada \\ Fernóndez, Ignacio Vidal García, fernando Bravo fleites y \\ Antonio Sánchez Sibello
}

\begin{abstract}
Resumen Se analizan los brotes de leptospirosis ocurridos en la provincia de Ciego de Ávila en el periodo de 1980 a 1995. En la etapa se notifican 40 brotes. Las actividades principales vinculadas a los mismos fueron la atención al cultivo de la caña de azúcar, al cultivo del plátano, el baño en río y las inundaciones. Se nota un incremento de brotes a partir del mes de junio. En los meses de octubre y noviembre se reportan las mayores incidencias. Los grupos de edades que más casos aportaron fueron de 10-14 años, 15-19 años y 30-34 años. El sexo más afectado fue el masculino. Los grupos más afectados fueron los estudiantes, pobladores urbanos y trabajadores agrícolas cañeros. De los 40 brotes, 21 fueron confirmados por medio de la prueba de microaglutinación y 19 por la prueba hemolitica, siendo los serogrupos más frecuentes Pomona y Australis.
\end{abstract}

Palabras-claves: Leptospirosis. Brotes. Estudio epidemiológico.

\begin{abstract}
Forty leptospirosis outbreaks occurred in Ciego de Avila province from 1980 to 1995. The main events involved in the outbreaks were floods, bathing in rivers and activities related to sugar cane and banana cultivation. The number of cases increased after June, with higher incidences reported in October and November. The most affected age groups were 10-14 years, 15-19 years and 30-34 years. Men were more affected than women. Students, people residing in the urban zone and farmers were the most affected groups. A total of 21 outbreaks out of 40 were confirmed by the microagglutination test and the remaining 19 by the hemolytic test. Pomona and Australis were the serogroups most frequently detected by microagglutination.
\end{abstract}

Key-words: Leptospirosis outbreaks.

La leptospirosis es una zoonosis bacteriana producida por el género leptospira. Dentro de las especies patógenas para el hombre se encuentran la Leptospira interrogans (complejo integrado por 24 serogrupos y 250 serovares) lo cual trae como consecuencia diversidad de cuadros clínicos y epidemiología diferente10 18.

Actualmente se señala que la distribución de estos gérmenes está condicionada por los llamados geofactores, los cuales son: climáticométrico, edófico, geográfico, biótico ${ }^{12}$. Además de las condiciones ecológicas, los factores sociales y económicos son importantes 16 .

La leptospirosis humana puede aparecer en forma esporádica o en brotes epidémicos, por lo general éstos se originan por exposición a aguas contaminadas por la orina de animales infectados 15 .

Además de los expuestos profesionalmente, se ha observado un aumento en la incidencia de niños, estudiantes y amas de casa y se han reportado comunidades urbanas y suburbanas

Centro Provincial de Higiene y Epidemiología. Provincia de Ciego de Avila, Instituto de Medicina Tropical "Pedro Kouri". Ciudad de la Habana y Universidad de Ciego de Avila, Cuba

Dirección para correspondencia: Dr. Miguel Suárez Hernández. Serafín Sánchez 155 entre Onelio Hernández y Martí, CP 65200 Ciego de Avila 2, Cuba.

Recebido para publicação em 3/7/98. 
afectadas más frecuentes que en zonas rurales 8 .

También se han observado brotes epidémicos vinculados a desastres hídricos5.

Se han detectado brotes de leptospirosis vinculados a ríos, presas, lagunas, canales, altas precipitaciones con o sin inundaciones, contacto directo con animales, trabajo en terrenos bajos, labores que contactan con residuales sólidos y líquidos tanto en poblaciones autóctonas como en inmigrantes y turistas3 111214 .

En Cuba el primer brote reportado se comunicó en 1910 entre los trabajadores que construían el alcantarillado de la Habana. En 1964 se reportó un brote de 7 casos en pacientes que laboraban en la siembra de pangola en la provincia de Holguín. En 1979 se reportaron 2 brotes, uno en la provincia Granma, vinculado a una unidad reproductora porcina y el otro en labores agrícolas en la provincia de Guantánamo con 5 fallecidos. A finales de 1980 surgió un brote que afectó a 167 trabajadores dedicados al corte de la caña de azúcar con 4 fallecidos 10 .

En 1981 se establece en el país el programa de control y prevención de la leptospirosis22, mejorando con el transcurso de los años las actividades de vigilancia en general.

Entre 1981 y 1994 se han publicado diversos brotes de leptospirosis humana en la literatura cubana, pero, no hemos encontrado ningún estudio donde se realice una descripción epidemiológica de todos los brotes ocurridos en un territorio por lo cual decidimos efectuar la presente investigación.

\section{MATERIAL Y MÉTODOS}

La provincia Ciego de Ávila está incluida en la región central de Cuba. El clima es similar al resto del país, tiene bien diferenciadas sus dos estaciones, la de lluvia (mayo-octubre) con valores medios de precipitaciones entre 1000 y 1300 milímetros y la de seca (noviembre-abril) cuyos valores oscilan de 200 a $300 \mathrm{~mm}$.

El principal potencial económico es la agricultura, siendo los principales cultivos la caña de azúcar, cítrico, piña, viandas y hortalizas; la superficie cultivada es alrededor de 23.000 caballerías, de ésto se deriva que un importante grupo de personas trabajen permanente 0 temporalmente en las labores agrícolas.

Los rios de la provincia son poco caudalosos y existen micropresas y presas en diferentes regiones geográficas.

Para la realización del presente trabajo fueron revisadas las informaciones existentes en el centro provincial de higiene y epidemiología de la provincia de Ciego de Ávila.

Se trata de un estudio descriptivo donde se analizan los brotes ocurridos en el período

\section{Prevención.}

\section{RESULTADOS.}

En los 16 años se presentaron 40 brotes con 176 enfermos, los años de mayor presentación fueron 1994 con 9 y 1987 con 5, aunque no se encontraron diferencias, estadísticamente significativas, entre los años de estudio $(p=0,204)$ (Figura 1). comprendido entre 1980 a 1995, así como el número de enfermos, actividades vinculadas a los brotes, la presentación por meses del año, los grupos de edades y sexo, el análisis de los brotes según actividad realizada, y también el diagnóstico serológico de los casos.

Para establecer si existían diferencias entre los grupos de edades, años estudiados y entre las actividades vinculadas a los brotes, se aplicó la prueba de Kolmogorov-Smirnov (K-S) para una muestra y la prueba de $\chi^{2}$ para la comparación entre los sexos y entre los grupos ocupacionales con un nivel de significación igual a 0,05 .

Se consideró brote de leptospirosis a la ocurrencia de más de un caso vinculado a una fuente de infección común cuando se cumplía el período de incubación de 2 a 20 días.

Se consideró caso confirmado de leptospirosis el que tuviera seroconversiones en la prueba hemolítica o microaglutinación22, aceptándose ambos técnicas, como diagnósticos en el Programa de Control y

Las actividades que más brotes aportaron fueron la atención al cultivo de la caña de azúcar, atención al cultivo del plátano, baño en ríos y contacto con inundaciones (Tabla 1).

Al aplicar la prueba de K-S se encontraron diferencias, estadísticamente significativas, 


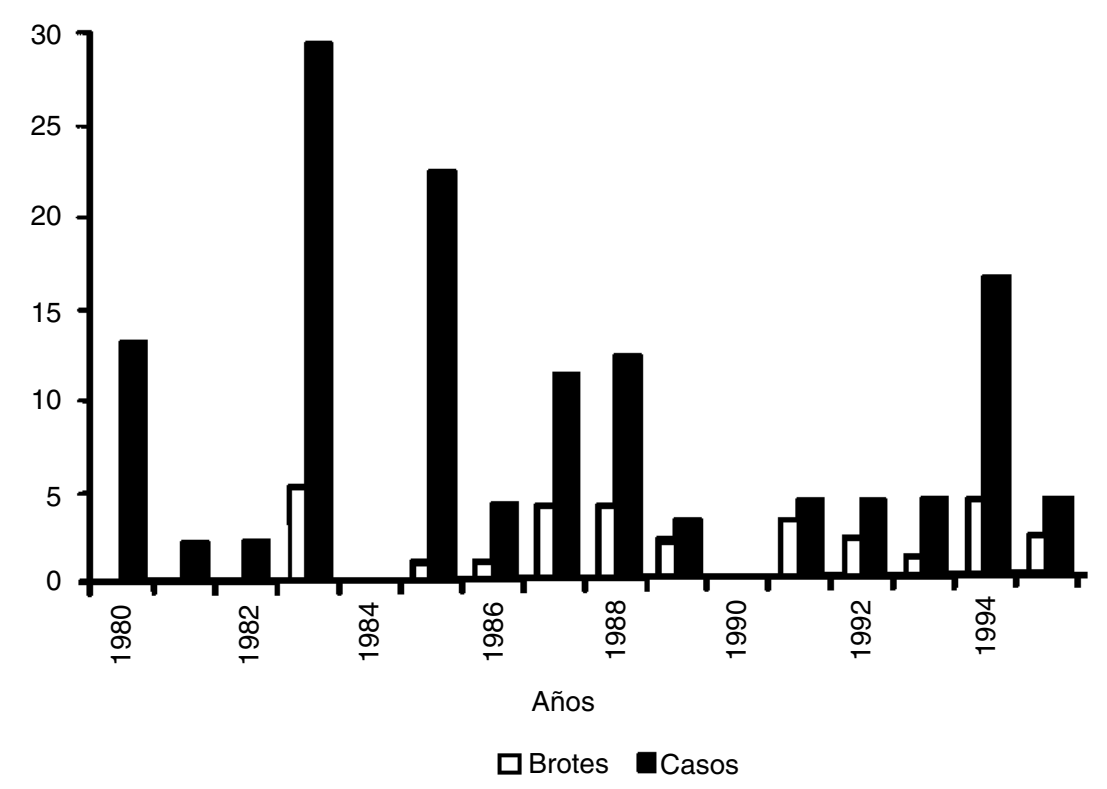
Figura 1 - Brotes y casos de leptospirosis humana. Provincia Ciego de Ávila, 1980-
1995.

Tabla 1 - Actividades vinculadas a los brotes.

\begin{tabular}{lcc}
\hline \multicolumn{1}{c}{ Actividades } & Número de brotes & Porciento \\
\hline Atención cultivo de la caña de azúcar & 8 & 20.0 \\
Atención cultivo del plátano & 5 & 12.5 \\
Baños en ríos & 5 & 12.2 \\
Relación con inundaciones & 5 & 12.5 \\
Baño en canales & 3 & 7.5 \\
Atención al cultivo del arroz & 3 & 7.5 \\
Baños en presas & 3 & 7.5 \\
Acuicultura & 2 & 2.0 \\
Contacto con terrenos bajos & 1 & 2.5 \\
Cría de terneros & 1 & 2.5 \\
Cría de equinos & 1 & 2.5 \\
Cría de perros & 1 & 2.5 \\
Atención al cultivo de la piña & 1 & \\
Recogida de naranja & 1 & \\
Total & 40 & \\
\hline
\end{tabular}

entre las actividades vinculadas a los brotes $(p=0,042)$.

En la Figura 2 aparecen los brotes por meses de presentación, en 10 de los 12 meses del año se presentaron brotes, octubre y noviembre tuvieron la mayor incidencia.

El $31 \%$ de los enfermos son niños y el $69 \%$ adultos, los grupos de edades de 10 a 14 años con el $24 \%$ seguido del de 15 a 19 años y 30 a 34 años con el 14\%, aportaron más casos, aunque no se encontraron diferencias, estadísticamente significativas, entre los grupos de edades estudiados $(p=0,86)$.

El $85 \%$ de los enfermos pertenecen al sexo masculino y el $15 \%$ al sexo femenino. Al aplicar la prueba de $\chi^{2}$ se encontraron diferencias estadísticamente significativas $\left(\chi^{2}=84,5\right.$; $\mathrm{p}<0,01$ ) (Tabla 2).

Los grupos que más brotes aportaron 


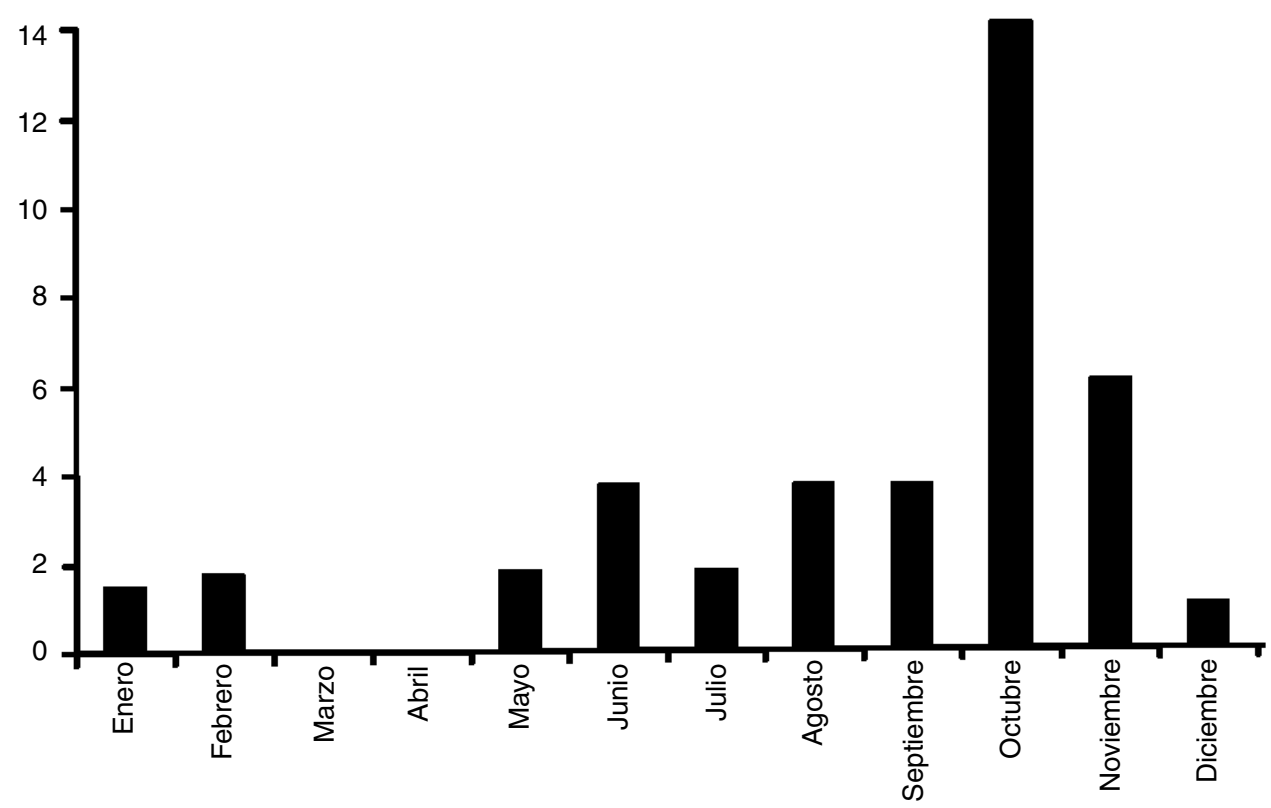

Figura 2 - Brotes de leptospirosis según meses del año, provincia Ciego de Ávila.

Tabla 2 - Grupos de edad y sexo de los casos confirmados.

\begin{tabular}{|c|c|c|c|c|}
\hline Grupos de edad & Masculino & Femenino & Total & $\%$ \\
\hline $0-4$ & 1 & 1 & 2 & 1.1 \\
\hline 5-9 & 6 & 4 & 10 & 5.7 \\
\hline $10-14$ & 35 & 8 & 43 & 24.4 \\
\hline $15-19$ & 24 & 1 & 25 & 14.2 \\
\hline $20-24$ & 24 & 0 & 24 & 13.7 \\
\hline $25-29$ & 13 & 0 & 13 & 7.4 \\
\hline 30-34 & 19 & 6 & 25 & 14.2 \\
\hline 35-39 & 6 & 4 & 10 & 5.7 \\
\hline $40-44$ & 10 & 1 & 11 & 6.2 \\
\hline$>44$ & 11 & 2 & 13 & 7.4 \\
\hline Total & 149 & 27 & 176 & 100 \\
\hline
\end{tabular}

Tabla 3 -Distribución de los brotes ocurridos según actividad realizada.

\begin{tabular}{lcr}
\hline Actividad realizada & № brotes & № casos \\
\hline Estudiantes & 9 & 41 \\
Pobladores urbanos & 6 & 23 \\
Trabajadores agricolas cañeros & 5 & 12 \\
Trabajadores temporales en cultivos varios & 4 & 24 \\
Trabajadores agrícolas en cultivos varios & 3 & 9 \\
Militares & 2 & 17 \\
Acuicultores & 2 & 6 \\
Sembradores temporales de arroz & 2 & 13 \\
Reclusos & 2 & 4 \\
Pescadores aficionados & 1 & 2 \\
Trabajadores temporales cañeros & 1 & 4 \\
Obreros pecuarios bovinos & 1 & 15 \\
Obreros pecuarios equinos & 1 & 2 \\
Salvamento & 1 & 4 \\
\hline Total & 40 & 176
\end{tabular}


fueron los estudiantes, pobladores urbanos y trabajadores agrícolas cañeros, existiendo diferencias, estadísticamente significativas, entre estas ocupaciones $(p<0,001)$ (Tabla 3 ).

Tabla 4 - Resultados serológicos según las pruebas utilizadas.

\begin{tabular}{|c|c|c|c|c|c|c|}
\hline \multirow[t]{2}{*}{ Prueba utilizada } & \multirow[t]{2}{*}{ Casos } & \multicolumn{5}{|c|}{ Serogrupos } \\
\hline & & Pomona & Australis & Canicola & Icterohaemorrhagiae & Ballum \\
\hline Microaglutinación & 21 & 8 & 6 & 4 & 2 & 1 \\
\hline Prueba hemolítica & 19 & * & * & * & * & * \\
\hline
\end{tabular}

* No procede.

De los 40 brotes, 21 se confirmaron por microaglutinación y 19 por prueba hemolítica, en los

primeros, los serogrupos de mayor incidencia fueron Pomona Australis y Canícola (Tabla 4).

\section{DISCUSIÓN}

Se ha estudiado el porqué, ante exposiciones iguales, en algunas situaciones se presentan casos esporádicos y en otras se notifican brotes, señalándose como factores la resistencia del huésped, las condiciones del medio ambiente y las características del agente 4 .

En los años de estudio, en nuestra provincia se presentaron un total de 40 brotes, sin embargo, en el período de 1991 a 1995 (5 años), se notificaron un total de 19 brotes que representa el $47 \%$ de los notificados en la etapa, esto se debe, en nuestra opinión que a partir de 1991 la provincia y el país comenzaron con serias dificultades económicas lo que provocó una disminución de las disponibilidades de rodenticida, dificultades con los medios de protección, suspensión de la vacunación anti-leptospira, por no suministro externo, entre 1992 y 1994 , incremento de la cría de cerdos, aumento de las siembras para autoabastecimiento e incremento de otras exposiciones de riesgo.

Es de señalar que aunque la provincia ha mantenido un trabajo sistemático desde el año 1985, ha perfeccionado en los últimos años su sistema de vigilancia epidemiológica.

El $50 \%$ de los brotes estuvieron vinculados a la agricultura, ésto se corresponde en gran medida con las bases económicas de la provincia, donde esta actividad es su pilar fundamental, sobre todo la producción azucarera que está entre las mayores del país.

En el período de junio a noviembre se notificaron 24 brotes coincidiendo con la época de lluvia, en los meses de julio y agosto los brotes están vinculados fundamentalmente a actividades recreativas motivado por el receso escolar y vacaciones, en esta época los brotes se presentaron fundamentalmente en niños y adultos jóvenes.

Con respecto a los grupos afectados vinculados a la agricultura fue similar la presentación de brotes en los expuestos permanentes que en los temporales. Algunos autores expresan que tienen menos posibilidad de enfermarse los que constantemente están expuestos, que los que lo hacen ocasionalmente, siendo el cuadro clínico más agresivo en estos7 13.

Diferentes autores han reportados brotes de leptospirosis. En la provincia de Ciego de Ávila, se reporta un brote de 21 casos en bañistas vinculado a un río y a una presa; presentándose 12 niños y 9 adultos predominando el serogrupo Australis 19. También en esta provincia se notifica un brote de 6 casos en niños a predominio meningoencefálico vinculado a un canal siendo el serogrupo Canicola el aislado en esta ocasión20.

Con respecto a las precipitaciones con o sin inundaciones se han reportado varios brotes. En Madrás (India), ocurrió un brote durante el monzón que afectó a humanos y perros21. En Costa Rica donde había poca información sobre leptospirosis ocurrió una epidemia después del paso del Huracán Juana en la ciudad de Cortés6.

Hay reportes de brotes vinculados al contacto directo con animales; en la Universidade de São

Paulo (Ribeirão Preto, SP, Brasil), se han notificado casos en manipuladores de animales de laboratorio17. En Argentina se describe un brote de leptospirosis que afectó a trabajadores de un 
matadero de bovinos y porcinos?.

\section{REFERENCIAS BIBLIOGRÁFICAS}

1. Agaev IA. The self maintenance of natural foci of leptospirosis. Zhurnal Mikrobiologil Epidemiologii Inmunobiologii 12:40-44, 1990.

2. Agaev IA. The mechanism maintaning epidemic foci of leptospirosis. Zhurnal Mikrobiologil Epidemiologii Inmunobiologii 2:38-41, 1991.

3. Andre-Fontaine G, Peslerbe X, Ganiere JP. Ocupational hazard of unnoticed leptospirosis in water ways maintenance staff. European Journal Epidemiology 8:228-232, 1992.

4. Atienzar E, Espino R, López C, Sed O, Alonso L. Brote de leptospirosis en la provincia de Camaguey y Las Tunas. Diagnóstico serológico, características clínicas y aislamiento microbiológico. Revista Cubana Medicina Tropical 37:105-112, 1985.

5. Biegel E, Mortensen H, Gaub J. Leptospirosis in the Rib Country 1980-1991. Ugeskrift for Laeger 157:157-161, 1995.

6. Boza R. Leptospirosis anicterica, análisis de una epidemia en Costa Rica. Acta Médica Costarricense 33:74-80, 1990.

7. Bren S, Radu O, Bauer T, Schonberg A, Reisshauer K, Waidman R, Kopp H. Leptospira infection rat population as probable cause of fatal case of Weil's disease. Berliner und Munchener Tierarztliche Wochenschrift 108: 405-407, 1995.

8. Cacciapuoti B, Ciceroni L, Pinto A, Apollini M, Rondinella V, Benedetti E, Cinco M, Dess J. Survey on the prevalence of leptospira infections in the Italian population. European Journal Epidemiology 10:173-180, 1994.

9. Caminoa R, Lapenta L, Gilardi R. Brote de leptospirosis humana en un matadero del Partido de Azul. Acta Bioquímica Clínica Latinoamericana 24:61-66, 1990.

10. Cruz R, López C, Sosa G, González M. Leptospirosis, salud del bovino y su repercusión en la producción animal y la salud pública. Editorial CIDA, La Habana, p:139, 1991.
11. Dai B. Advances in research on leptospira and human leptospirosis in China. Chinese Medical Society 7: 239243, 1992.

12. Ferguson TR. Leptospirosis surveillance 1990-1992. Communicable Disease Report 3:47-48, 1993.

13. Gendron Y, Prieur J, Gaufroy X, Gras C. Leptospirosis in French Polynesia 120 case reports. Medicine Tropicales 52: 21-27, 1992.

14. Jackson LA, Kaufman AF, Adams WG, Phelps MB, Andreasen C, Langkop C, Francis B, Wengerj W. J.D. Outbreak of leptospirosis associated with swimming. Pediatric Infectious Disease Journal 12:48-54, 1993.

15. Li Y. Cluster and multiple regression, analysis of leptospirosis epidemic factor. Chung Hua I Hsueh Tsa Chih (Chinese Medical Journal Taipei) 13:151-153, 1992.

16. Pereira M, Andrade J. Human leptospirosis in a slum area in the city of Rio de Janeiro, Brasil, a serological and epidemiological study. Memoria Instituto Oswaldo Cruz 85:47-52, 1990.

17. Pontes R, Girio R, Ventura A, Rufino N, Capuano D, Serfim A, Trivellato L, Romero E, Laus L. Surto de leptospiroses entre técnicos de laboratorio do Campus da Universidade de São Paulo de Ribeirão Preto, 1988. Medicina 23:169-178, 1990.

18. Sasaki DM, Pang L, Minette HP. Active surveillance and risk factors for leptospirosis in Hawaii. American Journal of Tropical Medicine and Hygiene 48:35-43, 1993.

19. Suárez M, Bustelo J, Gorvoy V, López C. Estudio epidemiológico de un brote de leptospirosis en el poblado de Jicotea de la provincia de Ciego de Avila. Revista Cubana Higiene y Epidemiología 27:272-274, 1989.

20. Suárez M, Bustelo J, Gorgoy V, Pérez L. Brote de leptospirosis en niños con predominio meningoencefálico en el municipio Morón. Revista Cubana Medicina Tropical 43:136-139, 1991.

21. Venkateraman KS, Nedunchelligan S. Epidemiology of outbreak of leptospirosis in man and dog. Comparative Inmunology Microbiology and Infectious Disease 15:243247, 1992.

22. Viceministerio de Higiene y Epidemiología. Programa de prevención y control de la leptospirosis en la República 